\title{
Time-frequency Features for Impedance Cardiography Signals During Anesthesia Using Different Distribution Kernels
}

\author{
Jesús Escrivá Muñoz ${ }^{1,2}$; Pedro Gambús ${ }^{3,4}$; Erik W. Jensen2; Montserrat Vallverdú1 \\ ${ }^{1}$ Biomedical Engineering Research Centre, CIBER of Bioengineering, Biomaterials and Nanomedicine (CIBER-BBN), \\ Universitat Politècnica de Catalunya, Barcelona, Spain; ${ }^{2}$ Quantium Medical, Mataró (Barcelona), Spain; ${ }^{3}$ Systems Phar- \\ macology Effect Control \& Modeling (SPEC-M) Research Group, Anesthesiology Department, Hospital CLINIC de \\ Barcelona, Barcelona, Spain; ${ }^{4}$ Department of Anesthesia and Perioperative Care, University of California San Francisco, \\ San Francisco, CA, USA
}

\begin{abstract}
Keywords
Time-frequency distributions, impedance cardiography, entropy, singular value decomposition
\end{abstract}

\section{Summary}

Objective: This works investigates the timefrequency content of impedance cardiography signals during a propofol-remifentanil anesthesia.

Materials and Methods: In the last years, impedance cardiography (ICG) is a technique which has gained much attention. However, ICG signals need further investigation. TimeFrequency Distributions (TFDs) with 5 different kernels are used in order to analyze impedance cardiography signals (ICG) before the start of the anesthesia and after the loss of consciousness. In total, ICG signals from one hundred and thirty-one consecutive patients undergoing major surgery under general anesthesia were analyzed. Several

Correspondence to:

Jesús Escrivá Muñoz

Quantium Medical

Av. Ernest Lluch 32

Office 3.17

08302 Mataró (Barcelona)

Spain

E-mail: jesus.escriva@upc.edu features were extracted from the calculated TFDs in order to characterize the time-frequency content of the ICG signals. Differences between those features before and after the loss of consciousness were studied. Results: The Extended Modified Beta Distribution (EMBD) was the kernel for which most features shows statistically significant changes between before and after the loss of consciousness. Among all analyzed features, those based on entropy showed a sensibility, specificity and area under the curve of the receiver operating characteristic above $60 \%$.

Conclusion: The anesthetic state of the patient is reflected on linear and non-linear features extracted from the TFDs of the ICG signals. Especially, the EMBD is a suitable kernel for the analysis of ICG signals and offers a great range of features which change according to the patient's anesthesia state in a statistically significant way.

Methods Inf Med 2018; 57(Open 1): e1-e9 https://doi.org/10.3414/ME17-01-0071 received: July 17, 2017

accepted: October 24, 2017

Funding

This work was supported by the Industrial PhD Program DI-2014/013 (Secretaria d'Universitats i Recerca del Departament d'Economia i Coneixement, Generalitat de Catalunya, Spain) and the European Social Fund of the European Union. CIBER of Bioengineering, Biomaterials and Nanomedicine is an initiative of ISCIII.

\section{Introduction}

In the last years, impedance cardiography (ICG) has proven to be an advantageous, inexpensive, non-invasive technique for monitoring the cardiovascular hemodynamic state of patients undergoing several medical procedures $[1,2,3,4,5,6,7]$. This work investigates the frequency content of the ICG signals in different moments of an anesthesia procedure: previous to the induction of anesthesia and after the start of an anesthesia procedure in the surgery room.

The spectral content of the ICG signals changes with time and thus time-frequency distributions (TFDs) are a convenient tool to analyze them. High-resolution time-frequency analysis is useful for signals which are nonstationary and/or multicomponent. TFDs is a technique which is often used in the case of analyzing electroencephalogram (EEG) $[8,9,10]$, and heart rate variability (HRV) $[11,12,13,14]$, amongst others. Any TFD application would ideally require high definition in spectral components, no cross-terms (in order to avoid confusing real components from artifacts or noise), a low computational complexity and some mathematical properties [15]. A considerable effort has been put into designing appropriate TFD depending on the characteristics of the signal to analyze $[10,16,17,18$, 19].

Nevertheless, TFDs contain considerably large amounts of data. Therefore, features are to be extracted from the TFDs in order to improve its characterization. Sev- 


\begin{tabular}{l|c|l|c}
\hline \multicolumn{2}{l}{ Patient Characteristics } & \multicolumn{2}{l}{ Medications } \\
\hline Age & $51.0 \pm 16.0$ years & Propofol & $131(100 \%)$ \\
\hline Height & $162.1 \pm 8.1 \mathrm{~cm}$ & Remifentanil & $131(100 \%)$ \\
\hline Weight & $68.2 \pm 12.8 \mathrm{~kg}$ & Rocuronium & $46(35.1 \%)$ \\
\hline LBM, Lean Body Mass & $47.7 \pm 7.7$ & Ephedrine & $4(3.1 \%)$ \\
\hline BSA, Body Surface Area & $1.73 \pm 0.21 \mathrm{~m}^{2}$ & Atropine & $21(16.0 \%)$ \\
\hline BMI, Body Mass Index & $26.0 \pm 4.7 \mathrm{~kg} / \mathrm{m}^{2}$ & & \\
\hline Gender (male/female) & $32 / 99$ & & \\
& $(24.4 \% / 75.6 \%)$ & & \\
\hline
\end{tabular}

eral authors have proposed different features applied to TFD in order to describe non-stationary signals or to locate events based on the signal entropy, energy concentration measures or singular values decomposition $[20,21,22,23]$. This work compiles some of those features in order to apply them to ICG signals. Several kernels including the Extended Modified Beta Distribution (EMBD) are also compared and discriminant analyses were conducted to differentiate between the TFD features from the ICG signals before and after the patient's loss of consciousness (LOC).

\section{Materials and Methods}

\subsection{Analyzed Data and Preprocessing}

One hundred and thirty-one consecutive patients undergoing major surgery under general anesthesia at the Hospital CLINIC de Barcelona (Spain) were assessed in this observational study. The details of the patients are reported in $>$ Table 1 . The patient characteristics included age, height, weight, lean body mass (LBM), body surface area (BSA), body mass index (BMI) and gender.

This observational study was conducted in compliance with the requirements of the Institutional Review Board and Ethics Committee of Hospital CLINIC de Barcelona (2013/8356) and adhered to the principles of the Declaration of Helsinki for medical research involving human subjects. All patients gave their written informed consent. Patients under eighteen years old or morbidly obese were excluded.

Propofol and remifentanil were administered. Anesthesia was induced with a tar-
Tab. 1

Patients' data and medications during surgical procedures. Qualitative data are presented as absolute frequencies and percentages; quantitative data are presented as mean \pm standard deviation.

get-controlled infusion system. The infusion rate of propofol was controlled by Schnider's pharmacokinetic model with 3 $\mu \mathrm{g} / \mathrm{ml}$ as effect-site target concentration and remifentanil was controlled by Minto's pharmacokinetic-pharmacodynamic model with $4 \mathrm{ng} / \mathrm{ml}$ as effect-site target concentration.

The impedance cardiography (ICG) was recorded by the qCO monitor (Quantium Medical, Spain) by using 4 electrodes, with one pair injecting a constant current (at 50 $\mathrm{kHz}$ ), and a second pair of electrodes measuring the resulting voltage. These signals are dimensionless and are recorded with a sampling rate of $250 \mathrm{~Hz}$.

This study aims to compare two anesthesia-related patient states: conscious and unconscious. During the induction of anesthesia, the moment of LOC was assumed to occur when patients lost response to verbal stimulation. To characterize each state, the ICG signal corresponding to a ten-second length taken 4 minutes after LOC (i.e., unconscious state or post-LOC state) was isolated and so was that corresponding to the ten seconds taken 4 minutes before LOC (i.e., conscious state or pre-LOC state). In the surgery room, ten seconds is a signal duration which generally ensures a quality recording without movement artifacts or other electrical noises.

\subsection{Analyzed Time-frequency Distributions}

Quadratic TFDs (QTFD) are based on estimating the instantaneous power spectrum of the signal, using a bilinear operator [24] and are the result of a trade-off between the cancelation of cross-terms and the frequency resolution. The Wigner-Ville dis- tribution (WVD) is the basic QTFD and is defined by taking the Fourier transform (FT) of an instantaneous auto-correlation function $K_{z}(t, \tau)$ described in Eq.(1).

$$
W_{z}(t, f)=\int_{-\infty}^{+\infty} K_{z}(t, \tau) e^{-2 j \pi f \tau} d \tau
$$

where $K_{z}(t, \tau)$ is defined as

$$
K_{z}(t, \tau)=z\left(t+\frac{\tau}{2}\right) z^{*}\left(t-\frac{\tau}{2}\right)
$$

and where $z(t)$ is the analytic associate of a real signal $x(t)$ obtained with the Hilbert transform $z(t)=x(t)+j H\{x(t)\}$.

Eq.(3) describes a general TFD as the convolution between the WVD and the 2D kernel $\gamma(t, f)$ formulated in the ambiguity domain such as $g(v, \tau)$ (where $v$ is Doppler and $\tau$ is lag). The WVD provides a highresolution representation of a signal in time and frequency but includes cross-terms in multicomponent signals. Therefore, the kernel used in the general formulation of the TFD reduces cross-terms although it also blurs auto-terms.

$$
\begin{aligned}
& \rho_{z}(t, f)=\gamma(t, f) \underset{(t, f)}{* *} W_{z}(t, f)= \\
& \int_{-\infty}^{\infty} \int_{-\infty}^{\infty} g(v, \tau) A_{z}(v, \tau) e^{j 2 \pi(v t-f \tau)} d v d \tau
\end{aligned}
$$

where $A_{z}(v, \tau)$ is the ambiguity function of the analytic associate of the real signal under analysis.

In this work, several TFD kernels have been used: the Choi-Williams Distribution (CWD), the Modified B-Distribution (MBD) and the Extended Modified B-Distribution (EMBD), the spectrogram with Hanning window, and the Zhao-AtlasMarks distribution (ZAM). Their parameters have been selected by optimizing the geometrical characteristics of the resulting TFDs of a synthetic ICG signal with known time-frequency (TF) parameters. The optimization was accomplished based on a former work by Sucic and Boashash [19]. The details of the kernels are included in - Table 2. 


\subsection{TFD-derived Parameters}

The time variation in the spectrum of a signal can be characterized with several features extracted from its TFDs. This paper analyses a collection of TFD-derived features based on singular value decomposition (SVD), entropy, extended time-.domain, energy concentration and sub-bands energy.

\subsubsection{SVD-based TFD-derived Features}

TFDs can be decomposed using its singular values in the form $\rho=U S V^{H}$, where $U$ is an $N \times N$ matrix, $S$ is an $N \times M$ diagonal matrix with positive real singular values $\sigma_{i}$, and $V^{H}$ is an $M \times M$ real unitary matrix. Following previous works [20,21, 22], in this investigation several features are extracted from the singular values $\sigma_{i}$ of the TFD, such as: $F_{S V D 1}$, the maximum $\sigma_{i} ; F_{S V D 2}$, standard deviation of $\sigma_{i}$; and $F_{S V D 3}$, the number of non-zero $\sigma_{i}$.

\subsubsection{Entropy-based TFD-derived Features}

The concept of Shannon Entropy [26] has been applied to both the design of new TFDs with minimum entropy [23] and the quantification of TFD complexity in TFDs. If the TFD is interpreted as a quasi-probability distribution, a highly-concentrated TFD with a small number of components has a lower entropy than a signal with a large number of signal components. The TFD complexity (TFCM) in Eq.(4) uses both SVD and Shannon entropy concepts and it represents the magnitude and the number of the non-zero singular values of the TFD $[20,21]$. It is a useful feature as their magnitudes have a strong relationship with the information content in the TFD.

$$
T F C M=-\sum_{\mathrm{i}=1}^{\mathrm{N}} \bar{\sigma}_{l} \log \bar{\sigma}_{l}
$$

where $\bar{\sigma}_{l}$ are the $N$ normalized singular values, i.e.: $\bar{\sigma}_{l}=\sigma_{i} / \sum_{i=1}^{N} \sigma_{i}$.

If the entropy of a TFD is to be calculated without using its singular values, the Time-Frequency Rényi entropy (TFRE) in
Tab. 2

Kernels for the QTFDs [25] and their parameter values used for the ICG characterization. The parameters $a, \alpha, \beta$ and $\sigma$ and the window length $W$ define the kernel shape.

\begin{tabular}{l|l|l}
\hline TFD & Kernel $\mathbf{g}(\mathbf{v}, \mathbf{T})$ & Parameters \\
\hline CWD & $\mathrm{e}^{-v^{2} \tau^{2} / \sigma}$ & $\sigma=4.12$ \\
\hline MBD & $\frac{|\Gamma(\beta+j \pi v)|^{2}}{\Gamma^{2}(\beta)}$ & $\beta=0.0026$ \\
\hline EMBD & $\frac{|\Gamma(\beta+j \pi \tau)|^{2}}{\Gamma^{2}(\beta)} \frac{|\Gamma(\alpha+j \pi v)|^{2}}{\Gamma^{2}(\alpha)}$ & $\begin{array}{l}\alpha=0.002 \\
\beta=0.988\end{array}$ \\
\hline Spectrogram & $w(v+\tau / 2) w(v-\tau / 2)$ & Hanning, $W=919$ \\
\hline ZAM & $w(\tau) \frac{a}{2|\tau|} \operatorname{sinc}\left(\frac{2 v \tau}{a}\right)$ & $a=2.3$ \\
\hline
\end{tabular}

(5) is used in substitution of the Shannon entropy [27]. The latter cannot be used for the majority of TFDs as these are not nonnegative. TFRE is a statistical tool sensitive to the number of signal components, their time duration and bandwidth, and their amplitude ratios.

$$
\begin{aligned}
\operatorname{TFRE}_{q}= & \frac{1}{1-q} \log _{2} \sum_{\mathrm{n}=1}^{\mathrm{N}} \\
& \sum_{\mathrm{m}=1}^{\mathrm{M}} \rho^{q}[\mathrm{n}, \mathrm{m}], q>2
\end{aligned}
$$

The TFRE for odd values of $q$ causes zeromean cross-terms to diminish due to the summation operation. Thus, the TFRE cannot discriminate a high-resolution TFD with significantly reduced cross-terms from a high-resolution TFD without any suppression of cross-terms. The TFD normalized Rényi entropy (TFNRE) in Eq.(6) solves this issue so that cross-terms have an overall effect of reducing the TFNRE.

$$
\begin{gathered}
\operatorname{TFNRE}_{q}=-\frac{1}{2} \log _{2} \sum_{\mathrm{n}=1}^{\mathrm{N}} \sum_{\mathrm{m}=1}^{\mathrm{M}} \\
{\left[\frac{\rho[\mathrm{n}, \mathrm{m}]}{\sum_{\mathrm{n}=1}^{\mathrm{N}} \sum_{\mathrm{m}=1}^{\mathrm{M}}|\rho[\mathrm{n}, \mathrm{m}]|}\right]^{q}+\log _{2} \delta_{t} \delta_{f}}
\end{gathered}
$$

where $\delta_{t}$ and $\delta_{f}$ are the time and frequency sampling steps, respectively. Baranjiuk et al. $[28,29]$ analyzed the influence of the parameter $q$ when calculating both TFRE and TFNRE and concluded that non-integer orders are yield complex values and so appeared of limited utility (6). In this study, a large range of $q$ values $(q=3,4, \ldots, 14,15$, $18,21,24,27,30,35,40,45,50)$ have been selected for TFRE and TFNRE in order to analyze its influence.

\subsubsection{Extended Time-Domain TFD-derived Features}

In order to use statistical time-domain features, such as mean and variance, the onedimensional time-domain moments have

Tab. 3 Time-frequency extension of time-domain features.

\begin{tabular}{l|l|}
\hline Feature & TF Formulation \\
\hline TFD Mean & $m_{T F}=\frac{1}{N M} \sum_{\mathrm{n}=1}^{\mathrm{N}} \sum_{\mathrm{m}=1}^{\mathrm{M}} \rho[\mathrm{n}, \mathrm{m}]$ \\
\hline TFD Variance & $\sigma_{T F}^{2}=\frac{1}{N M} \sum_{\mathrm{n}=1}^{\mathrm{N}} \sum_{\mathrm{m}=1}^{\mathrm{M}}\left(\rho[\mathrm{n}, \mathrm{m}]-m_{T F}\right)^{2}$ \\
\hline TFD Skewness & $\gamma_{T F}=\frac{1}{(N M-1) \sigma_{T F}^{3}} \sum_{\mathrm{n}=1}^{\mathrm{N}} \sum_{\mathrm{m}=1}^{\mathrm{M}}\left(\rho[\mathrm{n}, \mathrm{m}]-m_{T F}\right)^{3}$ \\
\hline TFD Kurtosis & $k_{T F}=\frac{1}{(N M-1) \sigma_{T F}^{4}} \sum_{\mathrm{n}=1}^{\mathrm{N}} \sum_{\mathrm{m}=1}^{\mathrm{M}}\left(\rho[\mathrm{n}, \mathrm{m}]-m_{T F}\right)^{4}$ \\
\hline $\begin{array}{l}\text { TFD Coefficient of } \\
\text { variation }\end{array}$ & $c_{T F}=\frac{\sigma_{T F}}{m_{T F}}$ \\
\hline
\end{tabular}


Tab. 4 Selected features to distinguish between pre-LOC and post-LOC ICG segments. All features have arbitrary units.

\begin{tabular}{|c|c|c|}
\hline Class & Feature name & Formulation \\
\hline \multirow[t]{3}{*}{ SVD-based TFD-derived Features } & Maximum SV & $F_{S V D 1}$ \\
\hline & SV Standard Deviation & $F_{S V D 2}$ \\
\hline & SV Range & $F_{S V D 3}$ \\
\hline \multirow[t]{3}{*}{ Entropy-based TFD-derived Features } & TF Complexity & TFCM \\
\hline & TF Rényi Entropy & $\operatorname{TFRE}_{q}$ \\
\hline & TF Normalized Rényi Entropy & TFNRE $_{q}$ \\
\hline \multirow{5}{*}{$\begin{array}{l}\text { Extended Time-Domain TFD-derived } \\
\text { Features }\end{array}$} & Mean & $m_{T F}$ \\
\hline & Variance & $\sigma_{T F}^{2}$ \\
\hline & Kurtosis & $k_{T F}$ \\
\hline & Skewness & $V_{T F}$ \\
\hline & Coefficient of variation & $C_{T F}$ \\
\hline Energy Concentration & Energy Concentration & ECOME \\
\hline Sub-Bands Energy-based Features & Energy in $i$-th band & $F_{\text {Band }_{i}}, i=1 \ldots 138$ \\
\hline
\end{tabular}

been replaced with the corresponding twodimensional TF characteristics according to [30]. Table 3 includes all extended time-domain features.

$$
\operatorname{ECOME}=\left(\sum_{\mathrm{n}=1}^{N} \sum_{\mathrm{m}=1}^{M}|\rho[\mathrm{n}, \mathrm{m}]|^{\frac{1}{r}}\right)^{r}, r>1
$$

\subsubsection{Energy Concentration}

The energy concentration measure (ECOME) determines the concentration of the dominant component at each location in the TF domain. Signals with TFD distributed in the TF plane will have a larger ECOME, while concentrated TFDs will have a smaller ECOME. quency plane of the TFDs has been divided by visual inspection and by using pairs of logarithmically spaced values. In total, 138 frequency bands have been analyzed and their corresponding features have been calculated using Eq.(13).

$$
F_{\text {Band }_{i}}=\sum_{\mathrm{n}=1}^{N} \sum_{\mathrm{m}=M_{\mathrm{i} 0}}^{M_{\mathrm{i} 1}} \rho[\mathrm{n}, \mathrm{m}], i=1 \ldots 138
$$

where $M_{i 0}$ and $M_{i 1}$ are the starting and end frequencies of the $i$-th band. Most of the content of the ICG signals is in the band between $0.5 \mathrm{~Hz}$ and $4 \mathrm{~Hz}$. Therefore, the spectrum has initially been divided in 55 logarithmically-distributed partitions (i.e. a partition is a single value). Bands have then been defined: A. for each partition; B. for each group of two successive partitions; and $\mathrm{C}$. for the frequencies from $0 \mathrm{~Hz}$ until each partition.

\subsection{Selected Features and Statistical Analysis}

In order to characterize signals corresponding to the segment previous to the LOC and that after the LOC, a range of features were selected in our study. These features are listed in $>$ Table 4.

Statistical analyses were performed using SPSS (Version 24, IBM, USA) and MATLAB $^{\circ}$ (MathWorks, USA). Quantitative data are presented as mean \pm standard deviation and qualitative data as frequency (percentage). A non-parametric test, the Wilcoxon signed-rank test, was

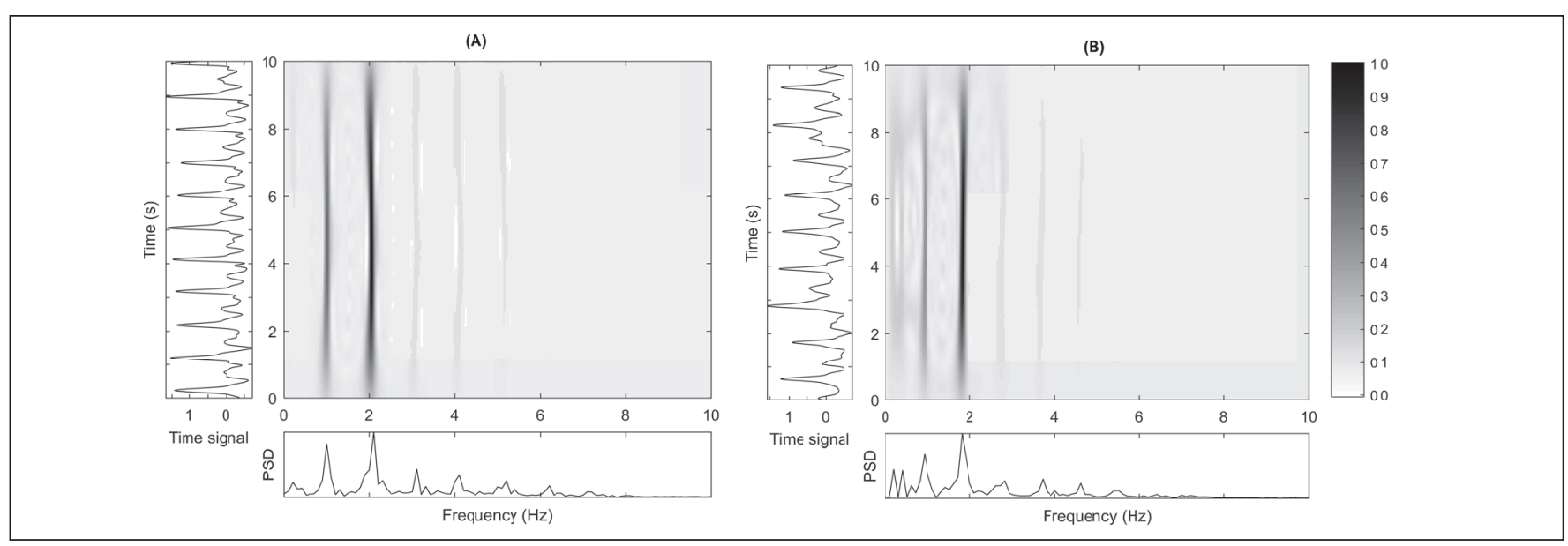

Fig. 1 EMBD of an ICG segment before LOC (A) and after LOC (B). 
Tab. 5 Mean and standard deviation of the SVD-based TFD parameters. All changes between pre-LOC and post-LOC are statistically significant. * indicates that Sen $>60 \%$, Spe $>60 \%$ and AUC $>60 \%$.

\begin{tabular}{l|l|l|l|l|l|l}
\hline & \multicolumn{2}{l}{$\boldsymbol{F}_{\text {SVD1 }}$} & \multicolumn{2}{l}{$\boldsymbol{F}_{\text {SVD2 }}$} & \multicolumn{2}{l}{$\boldsymbol{F}_{\text {SVD3 }}$} \\
\cline { 2 - 7 } & pre-LOC & post-LOC & pre-LOC & post-LOC & pre-LOC & post-LOC \\
\hline CWD & $(4.0 \pm 3.0) \cdot 10^{4}$ & $(2.4 \pm 1.6) \cdot 10^{4}$ & $(9.8 \pm 7.1) \cdot 10^{2}$ & $(5.6 \pm 3.7) \cdot 10^{2}$ & $(8.1 \pm 1.4) \cdot 10^{1}$ & $(7.4 \pm 1.3) \cdot 10^{1}$ \\
\hline MBD & $(1.5 \pm 1.1) \cdot 10^{5}$ & $(9.2 \pm 6.4) \cdot 10^{4}$ & $(3.6 \pm 2.6) \cdot 10^{3}$ & $(2.0 \pm 1.4) \cdot 10^{3}$ & $(1.3 \pm 0.1) \cdot 10^{2}$ & $(1.2 \pm 0.2) \cdot 10^{2}$ \\
\hline EMBD & $(1.4 \pm 1.1) \cdot 10^{7}$ & $(8.4 \pm 5.8) \cdot 10^{6}$ & $(3.2 \pm 2.3) \cdot 10^{5}$ & $(1.8 \pm 1.2) \cdot 10^{5}$ & $(2.4 \pm 0.6) \cdot 10^{2 *}$ & $(2.1 \pm 0.5) \cdot 10^{2}$ \\
\hline Spec. (Han.) & $(1.5 \pm 1.1) \cdot 10^{7}$ & $(8.3 \pm 5.6) \cdot 10^{6}$ & $(3.1 \pm 2.3) \cdot 10^{5}$ & $(1.7 \pm 1.1) \cdot 10^{5}$ & $(1.0 \pm 0.1) \cdot 10^{2}$ & $(9.7 \pm 1.2) \cdot 10^{1}$ \\
\hline ZAM & $(6.3 \pm 4.7) \cdot 10^{6}$ & $(3.8 \pm 2.7) \cdot 10^{6}$ & $(1.6 \pm 1.2) \cdot 10^{5}$ & $(9.1 \pm 6.0) \cdot 10^{4}$ & $(5.4 \pm 1.9) \cdot 10^{2}$ & $(4.6 \pm 1.4) \cdot 10^{2}$ \\
\hline
\end{tabular}

used to investigate whether the analyzed features changed after induction of anesthesia. Features that satisfy this condition were considered for building a linear discriminant function. The leave-one-out method was used for validation. Sensitivity (Sen), specificity (Spe) and the area under (AUC) the Receiver operating characteristic (ROC) curve were calculated to assess the ability of the studied features to predict the occurrence of LOC. Sen represents the proportion of pre-LOC ICG segments correctly classified and Spe represents the proportion of post-LOC ICG segments correctly classified. Grouped sensitivities and specificities are presented as mean [95\% confidence interval (CI)]. In the classification, the cut-off values are always the main of the centroids of the groups. Predicted membership is calculated by first producing a discriminant score for each case using a linear discriminant function. Then cases are classified in a concrete group depending on whether their discriminant score is smaller or larger than the cut-off value. Relationship between time-frequency derived indices and patient characteristics was assessed using Pearson's coefficient of correlation $(\rho)$. Significance level is always set at $\mathrm{p}<0.05$.

\section{Results}

After isolating ten-second segments from before and after the LOC, TFDs were calculated with different kernels and, then, the features were extracted and analyzed. - Figure 1 displays an example of a case analyzed using an EMBD. Figure 1A shows the pre-LOC TFD and Figure $1 \mathrm{~B}$ shows the post-LOC TFD. The main differences between the two states are the content below $1 \mathrm{~Hz}$ and the instantaneous frequencies, which seem to be lower in $>$ Figure $1 \mathrm{~B}$. The subsequent results aim to show tables and figures how the several TFDderived Features reported in $>$ Table 4 are related to the patient's state.

\subsection{SVD-based TFD-derived Features}

SVD-based TFD features change from before to after the LOC in a statistically significant manner in the case of $F_{S V D 1}, F_{S V D 2}$ and $F_{S V D 3}$ values. Table 5 shows the average values for these features for all the TFD kernels. It can be seen that the defined SVD features are higher before LOC than after LOC. These results have been obtained with values of Sen, Spe and AUC very similar for all kernels and all SVDbased TFD features. In this way, over the different kernels used, $\operatorname{Sen}(\%)$ is 76.4 $[75.3,77.5]$ for $F_{S V D 1}, 78.0[77.4,78.6]$ for $F_{S V D 2}$ and $65.0[61.8,68.2]$ for $F_{S V D 3} . S p e(\%)$ is $49.2[48.8,49.6]$ for $F_{S V D 1}, 51.0[50.6,51.4]$ for $F_{S V D 2}$ and $57.0[54.5,59.5]$ for $F_{S V D 3}$.

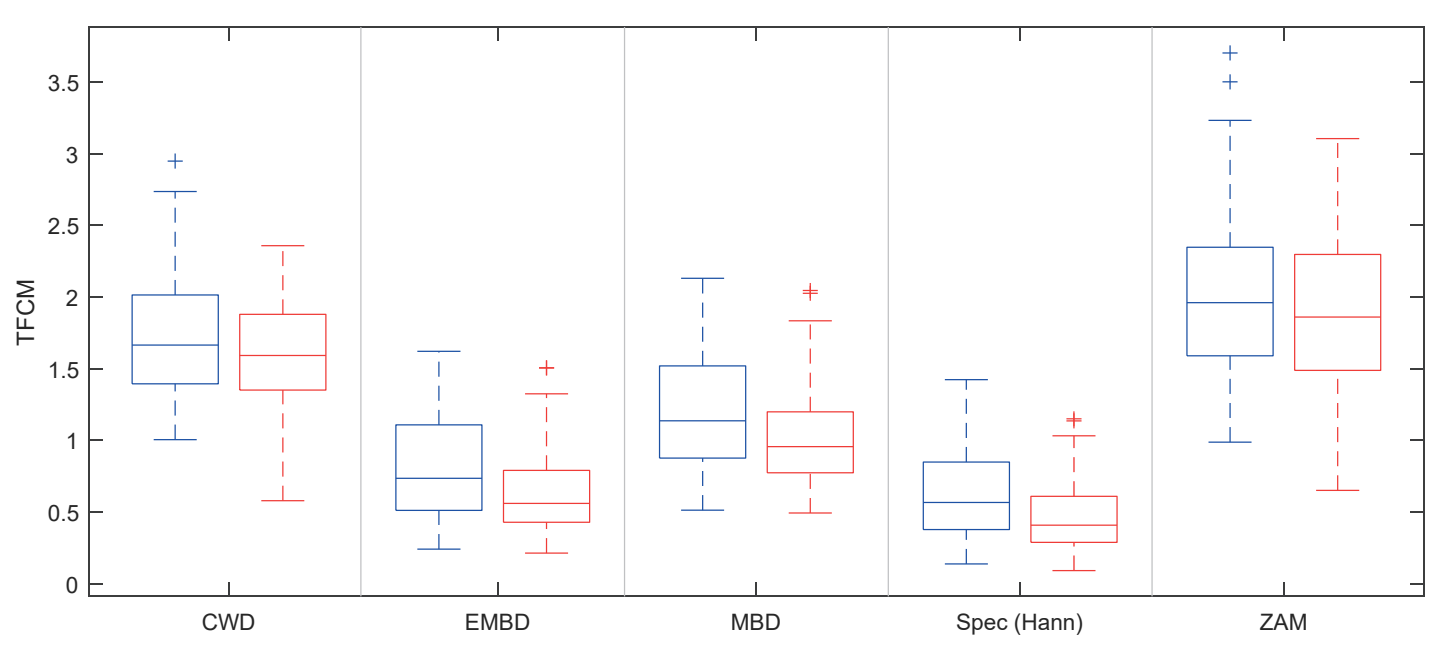

Fig. 2 TFCM values for different kernels before (blue) and after (red) LOC. All changes are statistically significant. 

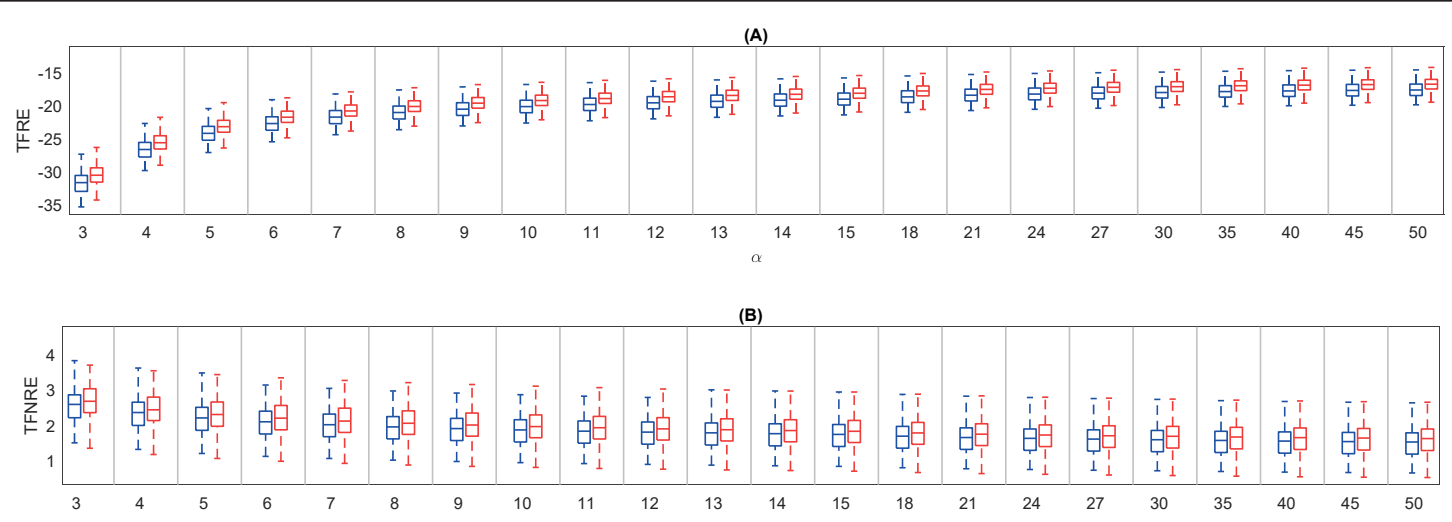

Fig. 3 Pre (blue) and post-LOC (red) median values for TFRE (A) and TFNRE (B) for the EMBD. Changes between pre and post LOC values are always statistically significant $(p<0.05)$.

AUC is $0.69[0.68,0.70]$ for $F_{S V D 1}, 0.70$ $[0.70,0.70]$ for $F_{S V D 2}$ and $0.65[0.64,0.66]$ for $F_{S V D 3}$. The best SVD-based TFD feature is $F_{S V D 3}$ calculated with an EMBD kernel, which presents an AUC $=0.63$, Sen $=$ $67.7 \%$ and $S p e=60.3 \%$.

\subsection{Entropy-based TFD-derived Features}

Regarding the entropy-based TF features, several results have been obtained. The TFCM presents statistically significant differences between pre-LOC and post-LOC for all kernels (see 2). In average for all kernels, $\operatorname{Sen}(\%)$ is $65.4[60.9,69.9]$, Spe $(\%)$ is $51.6[50,53.2]$ and AUC is $0.62[0.59,0.65]$. The complexity of the TFD responses is greater during pre-LOC than during postLOC.

$T_{F R E}$ always shows statistically significant differences between pre-LOC and
post-LOC for all kernels and for all $q$ values. Furthermore, Sen, Spe and AUC are similar for all $q$ values as seen in $>$ Figure 3 and also for all kernels as $>$ Table 6 shows. - Figure $3 \mathrm{~A}$ shows the $T F R E_{q}$ for an exemplary kernel such as the EMBD for all the different $q$ values. TFRE $E_{q}$ emphasizes high probabilities when $\mathrm{q}>1$. This figures shows how the values converge as the $q$ increases and the values are always higher for the pre-LOC signals than for the post-LOC signals.

TFNRE also shows statistically significant differences between pre and post-LOC for all $q$ values in the case of the spectrogram and in the case of the EMBD for $q \geq$ 6. Compared to the TFRE values, the normalization has decreased the AUC below 0.6 in all cases and Sen and Spe are below $60 \%$. Figure $3 \mathrm{~B}$ also shows the TFNRE for an exemplary kernel such as the EMBD for all the different $q$ values.
Tab. 6 Mean $[95 \% \mathrm{Cl}]$ of area under the curve (AUC) of the receiving operating curve (ROC), sensitivity (Sen) and specificity (Spe) of the $T R F E_{q}$ feature for all the studied distributions and all q values. $T R F E_{50}$ values for before and after the LOC have also been included. $\mathrm{Cl}$ of AUC are the same as the mean and thus are not included.

\begin{tabular}{ll|l|l|l|l}
\hline & Sen (\%) & Spe (\%) & AUC & $\begin{array}{l}\text { pre-LOC } \\
\text { TFRE }_{50}\end{array}$ & $\begin{array}{l}\text { post-LOC } \\
\text { TFRE }_{50}\end{array}$ \\
\hline CWD & $61.52[61.35,61.69]$ & $68.90[68.64,69.16]$ & 0.69 & $-9.26 \pm 1.18$ & $-8.51 \pm 1.13$ \\
\hline MBD & $61.07[60.86,61.27]$ & $67.17[66.66,67.69]$ & 0.69 & $-11.38 \pm 1.25$ & $-10.59 \pm 1.21$ \\
\hline EMBD & $63.01[62.91,63.11]$ & $69.33[69.04,69.62]$ & 0.71 & $-17.64 \pm 1.24$ & $-16.77 \pm 1.20$ \\
\hline Spec. (Han) & $62.29[61.90,62.67]$ & $69.92[69.69,70.14]$ & 0.72 & $-17.15 \pm 1.23$ & $-16.22 \pm 1.13$ \\
\hline ZAM & $62.44[62.16,62.73]$ & $69.41[68.92,69.90]$ & 0.70 & $-16.73 \pm 1.22$ & $-15.91 \pm 1.15$ \\
\hline
\end{tabular}

\subsection{Extended Time-Domain TFD-derived Features}

The time-extended TF features show statistically significant differences between the pre-LOC and post-LOC values of $m_{T F}$ and $\sigma_{T F}^{2}$, for all TFD kernels. In addition, there are also statistically significant differences in the case of $k_{T F}$ for all TFD kernels except for the CWD and in the case of the $\gamma_{T F}$ for all TFD kernels except for CWD and ZAM. The difference between the pre-LOC and post-LOC values of $c_{T F}$ is only statistically significant for the spectrogram. For all TFD kernels, the AUC for these features is 0.70 for $m_{T F}$ and $\sigma_{T F}^{2}$, and between 0.51 and 0.63 for $k_{T F}, \gamma_{T F}$ and $c_{T F}$. Spe is always lower than $60 \%$ (between 38.2 and 54.2) for all time-extended TF features and for all kernels but $\operatorname{Sen}(\%)$ is in average 75.5 [75.2,75.8] for $m_{T F}$ and 85.8 [85.2,86.1] for $\sigma_{T F}^{2}$. Table 7 shows that all the time-ex-

Tab. 7 Time-extended TF features of the ICG signals before and after the LOC using the spectrogram kernel with a Hanning window. All changes are statistically significant. Features have arbitrary units.

\begin{tabular}{l|c|c|}
\hline & pre-LOC & post-LOC \\
\hline$m_{\text {TF }}$ & $486.5 \pm 340.5$ & $275.0 \pm 168.4$ \\
\hline$\sigma^{2} T F$ & $(3.6 \pm 5.3) \cdot 10^{7}$ & $(1.0 \pm 1.5) \cdot 10^{7}$ \\
\hline$k_{T F}$ & $16.1 \pm 2.8$ & $15.0 \pm 2.4$ \\
\hline$Y_{T F}$ & $319.0 \pm 100.9$ & $278.0 \pm 87.1$ \\
\hline$C_{T F}$ & $9.8 \pm 1.5$ & $9.5 \pm 1.3$ \\
\hline
\end{tabular}




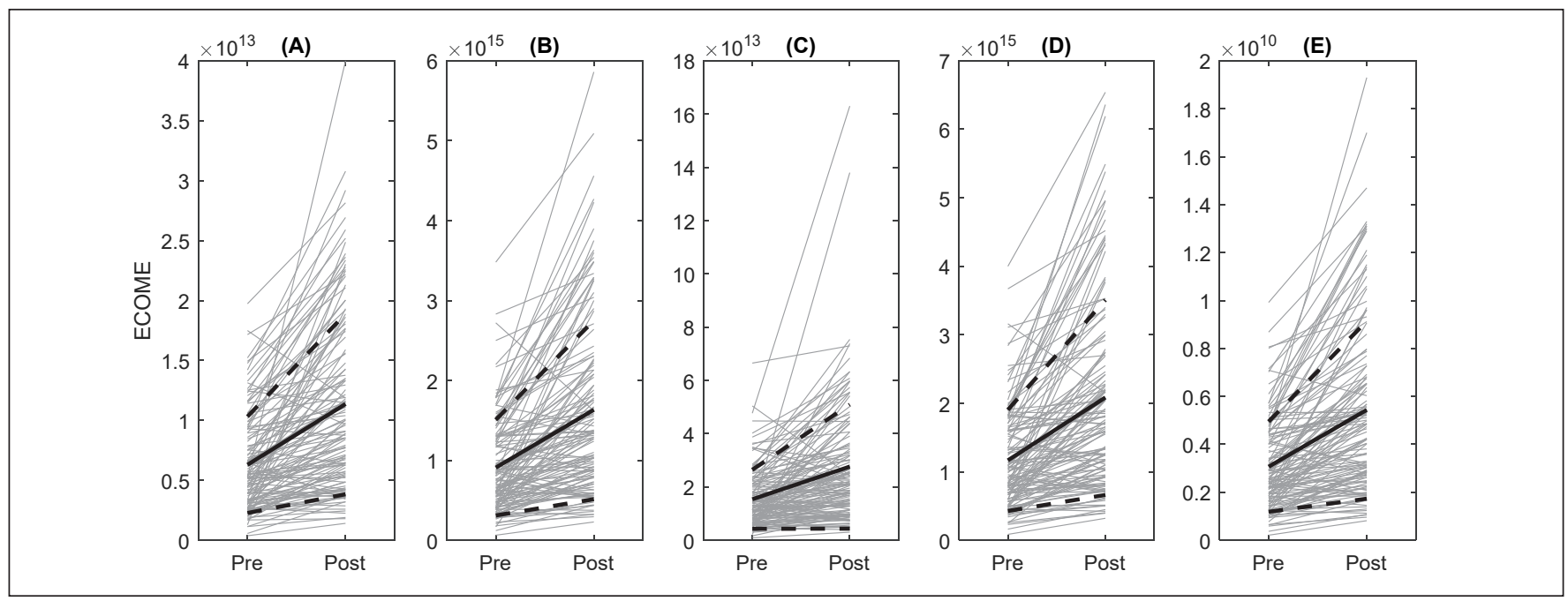

Fig. 4 Pre and post-LOC ECOME values for the CWD (A), the EMBD (B), the MBD (C), the spectrogram with a Hamming window (D) and the ZAM distribution (E). Changes between pre and post LOC values are always statistically significant $(p<0.05)$.

tended TF features decrease after LOC for the spectrogram. This also occurs for the rest of kernels.

\subsection{Energy Concentration}

ECOME values for all TFD kernels before and after LOC are plotted in $>$ Figure 4 and these are higher after the LOC than before it. All changes have proven to be statistically significant. In average, Sen is 75.3 [73.2,77.4], Spe is $51.6[49.9,53.3]$ and AUC is 0.72 for all kernels.

\subsection{Sub-Bands Energy-based Features}

The spectrum of the TFDs has been divided into 138 different frequency bands. The
MBD and the ZAM distribution are the ones with the largest number of statistically significant frequency bands, with 114 and 116 , respectively. The rest of kernels provide less significant bands: EMBD (106), CWD (100) and the spectrogram with a Hanning window (91). The spectral content of the TFD bands is always greater before the LOC than after the LOC. AUC is in almost all cases above 0.6 but both Sen and Spe are not larger than $60 \%$ at the same time. - Figure 5 shows how the energy in some of the frequency bands changes between before and after the LOC. Moreover, this figure also shows how most energy is concentrated between 1 and $4 \mathrm{~Hz}$. The very low frequency from 0 to $0.05 \mathrm{~Hz}$ is also prominent, due to the non-zero signal mean.

\section{Discussion}

TFDs have been analyzed using five different kernels and information has been extracted using several features based on SVD decomposition, entropy, extended time-domain, concentration and subbands energy. All features decreased after the LOC. The EMBD kernel offered the largest quantity of features with statistically significant differences (156). In total, 129 were found for CWD, 129 for MBD, 147 for the spectrogram and 146 for ZAM distribution. After EMBD, kernels such as the spectrogram and the ZAM distribution also offer a large amount of significant features but ZAM usually introduces more cross-terms than other distributions.
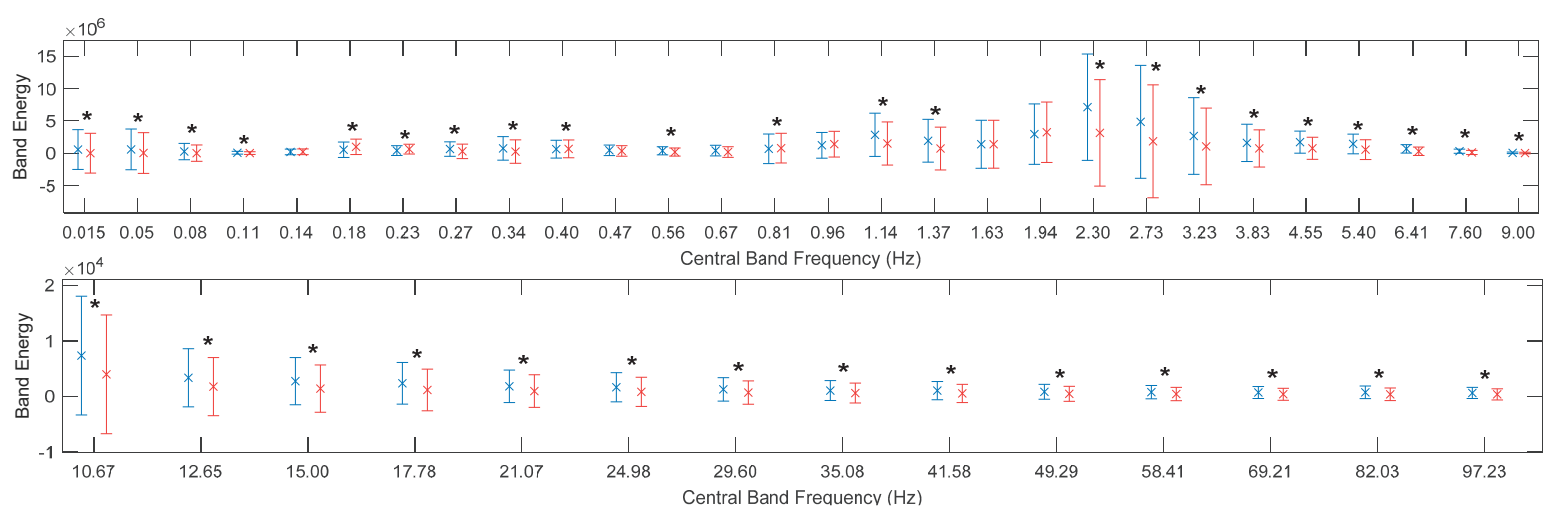

Fig. 5 Mean and standard deviation of the energy of some bands for the pre-LOC (blue) and post-LOC (red) periods. The kernel used for this figure is the MBD. * indicates that the change is statistically significant $(p<0.05)$. 
The robustness of the spectrogram is generally related to the lack of undesirable artifacts present in other TFDs since the non-linearity is introduced in the final step of the spectrogram computation. Nonetheless, the spectrogram does not satisfy the instantaneous frequency criterion of the quadratic class of TFDs and hence it does not allow the exact extraction of the signal IFs from its dominant peaks.

Among all the features which have been analyzed, TFRE is the most successful. For all kernel types and for any $q$ value, TFRE values decrease after the loss of consciousness and both their sensitivity and specificity are always above $60 \%$. Moreover, the AUC is always above 0.6 . The increase in the TFRE is theoretically related to the decrease of predictability or the increase of disorder. From a biological point of view, this would imply that the ICG signals are more deterministic after the LOC. Regarding the sub-bands energy-based features, these show that most of the ICG energy is concentrated between 1 and $4 \mathrm{~Hz}$, since their values are higher than in the rest of frequency band. Nevertheless, sensitivity and specificity of the features should be improved in the future for such features to be adequate for clinical applications.

Our study presents some limitations which must be considered. The pharmacological effects of the drugs infused in the patients may vary depending on the target concentrations. This is especially true when analysing signals after the LOC. This fact does not reduce the validity of results but should be taken into account especially in future works for which information from depth-of-anaesthesia monitors should be included.

\section{Conclusion}

In conclusion, this work presents a collection of various features which can be obtained from TFDs. Different kernel TFDs have been calculated and their results have been compared. When analyzing signals representing different anesthetic states, the TF Rényi entropy is the most prominent feature. Regarding the various kernels which have been analyzed, the EMBD is the most successful for the extraction of features showing statistically significant differences in different anesthesia points.

\section{Author Contribution}

J Escrivá Muñoz did the data base analysis and writing of the manuscript, and P Gambús collected the cases for the data base; EW Jensen and M Vallverdú act as academic advisors in the corresponding author's $\mathrm{PhD}$ program.

\section{Conflict of Interest}

J Escrivá Muñoz and EW Jensen work for Quantium Medical, SL.

\section{References}

1. Saugel B, Cecconi M, Wagner JY, Reuter DA. Noninvasive continuous cardiac output monitoring in perioperative and intensive care medicine. $\mathrm{Br} \mathrm{J}$ Anaesth 2015; 114(4): 562-575.

2. Bundgaard-Nielsen M, Ruhnau B, Secher NH, Kehlet $\mathrm{H}$. Flow-related techniques for preoperative goal-directed fluid optimization. Br J Anaesth 2007; 98(1): 38-44.

3. Truijen J, van Lieshout JJ, Wesselink WA, Westerhof BE. A recent meta-analysis comprehensively. J Clin Monit Comput 2012; 26(4): 267-278.

4. Rivers E, Nguyen B, Havstad S, Ressler J, Muzzin A, Knoblich B, Peterson E, Tomlanovich M. Early goal-directed therapy in the treatment of severe sepsis and septic shock. N Engl J Med 2001; 345(19): 1368-1377.

5. Gan TJ, Soppitt A, Maroof M, El-Moalem H, Robertson KM, Moretti E, Dwane P, Glass PSA. Goaldirected intraoperative fluid administration reduces length of hospital stay after major surgery. Anesthesiology 2002; 97(4): 820-826.

6. Siedlecka J, Siedlecki P, Bortkiewicz A. Impedance cardiography - Old method, new opportunities. Part II. Testing of cardiovascular response to various factors of work environment. Int J Occup Med Environ Health 2015; 28(1): 34-41.

7. Siedlecka J, Siedlecki P, Bortkiewicz A. Impedance cardiography - Old method, new opportunities. Part I. Clinical applications. Int J Occup Med Environ Health 2015; 28(1): 27-33.

8. Boashash B, Mesbah M. A time-frequency approach for newborn seizure detection. IEEE Eng Med Biol Mag 2001; 20(5): 54-64.

9. Stevenson N, Mesbah M, Boashash B. Quadratic time-frequency distribution selection for seizure detection in the newborn. In: IEEE Engineering in Medicine and Biology Society Conference. 2008. p. 923-926.

10. Boashash B, Ali N, Ben-jabeur T, Khan NA, Benjabeur T. Time - frequency features for pattern recognition using high-resolution TFDs: A tutorial review. Digit Signal Process 2015; 40: 1-30.

11. Huang HH, Chan HL, Lin PL, Wu CP, Huang CH. Time-frequency spectral analysis of heart rate variability during induction of general anaesthesia. Br J Anaesth 1997; 79: 754-758.

12. Chan H-L, Huang H-H, Lin J-L. Time-Frequency Analysis of Heart Rate Variability During Transient Segments. Ann Biomed Eng 2001; 29(11): 983-996.

13. Aimie-Salleh N, Malarvili MB, Phillip AC. Quantitative Comparison of Time Frequency Distribution for Heart Rate Variability Using Performance Measure. Journal of Wireless Networking and Communications 2015; 5: 1-5.

14. Orini M, Bailón R, Mainardi L, Laguna P. Synthesis of HRV signals characterized by predetermined time-frequency structure by means of timevarying ARMA models. Biomed Signal Process Control 2012; 7(2): 141-150.

15. Hlawatsch F, Flandrin P. The Interference Structure of the Wigner Distribution and Related TimeFrequency Signal Representation. In: Mecklenbräuker W, Hlawatsch F, editors. The Wigner Distribution - Theory and Applications in Signal Processing. Elsevier Science; 1997 p. 59-133.

16. Boashash B, Sucic V. Resolution measure criteria for the objective assessment of the performance of quadratic time-frequency distributions. IEEE Trans Signal Process 2003; 51(5): 1253-1263.

17. Ben-Jabeur T, Kadri A. Design of a New HighEnergy Concentration Kernel Quadratic TFD for EEG Spike Signal. In: 23rd European Signal Processing Conference. 2015. p. 2606-2610.

18. Abed M, Belouchrani A, Cheriet M, Boashash B. Time-frequency distributions based on compact support kernels: Properties and performance evaluation. IEEE Trans Signal Process 2012; 60(6): 2814-2827.

19. Sucic V, Boashash B. Optimisation algorithm for selecting quadratic time-frequency distributions: Performance results and calibration. In: 6th International Symposium on Signal Processing and Its Applications, ISSPA 2001. 2001. p. 331-334.

20. Boashash B, Boubchir L, Azemi G. A methodology for time-frequency image processing applied to the classification of non- stationary multichannel signals using instantaneous frequency descriptors with application to newborn EEG signals. J Adv Signal Proccessing 2012; 117: 1-21.

21. Fani M, Azemi G, Boashash B. EEG-based automatic epilepsy diagnosis using the instantaneous frequency with sub-band energies. In: 7th International Workshop on Systems, Signal Processing and their Applications (WOSSPA). 2011. p. 187-190.

22. Boashash B, Boubchir L, Azemi G. Improving the classification of newborn EEG time-frequency representations using a combined time-frequency signal and image approach. In: 2012 11th International Conference on Information Science, Signal Processing and their Applications (ISSPA). IEEE; 2012 p. 280-285.

23. Aviyente S, Williams WJ. Minimum entropy timefrequency distributions. IEEE Signal Process Lett 2005; 12 (1): 37-40.

24. Boashash B. Time-Frequency Signal Analysis and Processing: a Comprehensive Reference. Elsevier; 2003.

25. O’Toole J, Mesbah M, Boashash B. A Computationally Efficient Implementation of Quadratic Time-Frequency Distributions. In: 9th Inter- 
national Symposium on Signal Processing and Its Applications - ISSPA. 2007.

26. Shannon C, Weaver W. The Mathematical Theory of Communication. Urbana, Illinois: University of illinois Press; 1964.

27. Williams WJ, Brown ML, Hero AO. Uncertainty, information and time-frequency distributions. Proc. SPIE Int Soc Opt Eng 1991; 1566: 144-156.
28. Baraniuk RG, Flandrin P, Janssen AJEM, Michel OJJ. Measuring time-frequency information content using the Renyi entropies. IEEE Trans Inf Theory 2001; 47 (4): 1391-1409.

29. Flandrin P, Baraniuk RG, Michel O. Time-Frequency Complexity and Information. In: Proceedings of ICASSP '94. IEEE International Conference on Acoustics, Speech and Signal Processing. IEEE; 1994.

30. Boashash B, Azemi G, Khan NA, Ali Khan N Principles of time-frequency feature extraction for change detection in non-stationary signals: Applications to newborn EEG abnormality detection. Pattern Recognit 2014; 48 (3): 616-627. 